\title{
ANALYSIS OF BLOOD TRANSCRIPTOME IN PATIENTS WITH CHRONIC KIDNEY DISEASE OF UNCERTAIN AETIOLOGY
}

\author{
${ }^{1}$ S. Sayanthooran, ${ }^{1 *}$ D. N. Magana-Arachchi, ${ }^{2}$ L. Gunarathne, ${ }^{3}$ T. Abeysekera and
}

\author{
${ }^{4}$ S. D. S. S. Sooriyapathirana \\ ${ }^{I}$ National Institute of Fundamental Studies, Sri Lanka \\ ${ }^{2}$ Renal Care \& Research Centre, District Hospital, Girandurukotte, Sri Lanka \\ ${ }^{3}$ Department of Pharmacology, Faculty of Medicine, University of Peradeniya, Sri Lanka \\ ${ }^{4}$ Department of Molecular Biology and Biotechnology, University of Peradeniya, Sri Lanka
}

\begin{abstract}
Chronic kidney disease of uncertain aetiology $(\mathrm{CKDu})$ is an increasing health problem in certain agricultural regions of the tropical world including certain parts of Sri Lanka, Latin America, and India. This form of chronic kidney disease (CKD) does not have common causative factors such as diabetes or hypertension. The purpose of this study was to test the hypothesis that patients suffering with CKDu have differential expression patterns of genes in blood that will help differentiate them from healthy individuals, and to identify such differentially expressed genes as possible biomarkers of CKDu. Briefly, total RNA was isolated from peripheral whole blood of Stage 2, 3 and 4 CKDu patients in three different pools and compared to the expression pattern of a pooled sample of RNA extracted from healthy individuals. The RNA was amplified, reverse transcribed and hybridized to Illumina HumanHT-12 v4 Expression BeadChip arrays and scanned with an Illumina BeadArray Reader confocal scanner. Seven genes were identified that were commonly differentially expressed (fold change $\geq 2$ or $\leq 0.5$ ) in the three stages of CKDu population compared to healthy group. The genes identified included those involved in hypertensive response (ADM), gap junction channel activity (GJB4) and infectious/immune response (IFIT1, PI3, DEFA1, HBZ, RN7SK). These genes have the potential to be used as diagnostic markers of $\mathrm{CKDu}$ as they are differentially regulated in the studied stages of CKDu and can diagnose disease patients from the healthy individuals.
\end{abstract}

Keywords: Biomarkers, CKD, CKDu, gene expression analysis, peripheral whole blood, RT-qPCR

\section{INTRODUCTION}

Over a million people worldwide die every year due to end stage renal disease. The disease is an equal threat to both the developed and developing countries. Increased age contributes to the disease in developed countries whereas the lack of proper treatment and renal replacement and dialysis facilities are the major factor in developing countries [1]. Diagnosis of the disease is also complicated as symptoms become apparent only with worsening stages and it is usually too late for preventive measures.

The major concern in Sri Lanka is the rise of chronic kidney disease which is of unknown etiology
$(\mathrm{CKDu})$, and is currently threatening to reach epidemic proportions. This disease is mainly clustered in the North Central region of the country among the dry zones of the country. High incidence has been noted in the divisions of Medawachchiya, Girandurukotte, Mahiyanganaya, Padaviya, Medirigiriya, Dehiattakandiya, Nikawewa and Kebithigollewa [2].

CKDu is not only limited to Sri Lanka, but has been observed in other agricultural regions in the world. It has especially been noted in the cotton and sugarcane plantation workers in Central America and has been linked to the geographical location, where excessive heat and volume depletion are hypothesized to be the cause [3]. 
The disease is chronic tubulointerstitial in nature with the proximal tubules being mostly affected. There are multiple sources that have been implicated in other tubulointerstitial diseases including, but not limited to, toxins, drugs, cystic diseases, vascular conditions, infection, immunological conditions and mechanical damage. These conditions could also be playing a role in the tubulointerstitial pathology seen in chronic kidney disease of unknown etiology in Sri Lanka [2, 4].

Although the search for the possible cause of CKDu has been done from various different angles, few studies have looked at it from a molecular/genetic standpoint. Genome wide association studies and exome sequencing have been recently carried out and point to genetic susceptibility of patients $[5,6]$. Gene expression studies have also been carried out using selected panel of genes and have pointed towards environmentally induced oxidative stress and genetic susceptibility of individuals [7].

Gene expression analysis is a quantitative way of studying the expression of genes from the mRNA levels. Although all the genes are present in the genome, only some are expressed; the expression of which is regulated by complex mechanisms, both genetically and environmentally stimulated. The human gene expression varies significantly from individual to individual and the expression levels directly match with the underlying phenotype of the cells (Cheung \& Spielman, 2009). Expression patterns of genes can be both inherited and also modified by external environmental factors. It is found that factors such as diet or medication of individuals can have a significant influence on gene expression and therefore can even override the genetic influence on gene expression [8].

The research aimed to determine the gene expression patterns in blood of $\mathrm{CKDu}$ patients, to identify possible causative factor/s and biomarkers for the disease. By identifying commonly differentially expressed genes in the different stages of disease, a common biomarker for the disease can be identified.

\section{METHODOLOGY}

\section{Ethical clearance and informed consent}

Ethical clearance for the study was obtained from the relevant hospital authorities and the Postgraduate Institute of Science, University of Peradeniya, Sri Lanka. Written informed consent was obtained from each subject.

\section{Study Population}

Samples were collected in three batches for the three different stages of $\mathrm{CKDu}$; stage 2, stage 3 and stage 4, from August 2014 to June 2015. Six patients were recruited for the study from each of stage 2 , stage 3 and stage 4 of CKDu as diagnosed by the attending nephrologist from the Renal Clinic, District Hospital, Girandurukotte, a region of Sri Lanka endemic to $\mathrm{CKDu}$. Six healthy volunteers were also recruited for the study from the Kandy District, an area not endemic to $\mathrm{CKDu}$. Blood samples of $1 \mathrm{~mL}$ each was collected from the patients during routine blood collection.

\section{RNA Preparation}

Total RNA was extracted from whole blood using Qiazol (Qiagen, USA), purified using RNeasy columns (Qiagen, USA) according to the manufacturers' protocol. After processing with DNase digestion and clean-up procedures, RNA samples were checked for integrity on Agarose gel electrophoresis and quantified, aliquot and stored at $80^{\circ} \mathrm{C}$ until it was shipped to an external facility, Macrogen Inc., South Korea for microarray analysis.

\section{Microarray Procedures}

\section{Quality control}

For quality control of samples after shipment, RNA purity and integrity were evaluated by Macrogen Inc. (South Korea) using denaturing gel electrophoresis, OD 260/280 ratio, and analyzed on Agilent 2100 Bioanalyzer (Agilent Technologies, USA).

\section{Labeling and purification}

Total RNA was amplified and purified using the Ambion Illumina RNA amplification kit (Ambion, USA) to yield biotinylated cRNA according to the manufacturer's instructions. Briefly, $550 \mathrm{ng}$ of total 
RNA was reverse-transcribed to cDNA using a T7 oligo(dT) primer. Second-strand cDNA was synthesized, in vitro transcribed, and labeled with biotin-NTP. After purification, the cRNA was quantified using the ND-1000 Spectrophotometer (NanoDrop, USA).

\section{Hybridization and data export}

$750 \mathrm{ng}$ of labeled cRNA samples were hybridized to each human HT-12 expression v.4 bead array for 16$18 \mathrm{~h}$ at $58^{\circ} \mathrm{C}$, according to the manufacturer's instructions (Illumina, Inc., USA). Detection of array signal was carried out using Amersham fluorolink streptavidin-Cy3 (GE Healthcare Bio-Sciences, UK) following the bead array manual. Arrays were scanned with an Illumina bead array Reader confocal scanner according to the manufacturer's instructions

\section{Analysis of Microarray Data}

The results obtained from the primary microarray analysis were further analyzed. The genes that were differentially expressed (fold change $\geq 2$ or $\leq 0.5$ ) were selected from each of the three stages; stage 2 , stage 3 and stage $4 \mathrm{CKDu}$, and the genes common to all three stages were identified.

\section{RESULTS}

Differentially expressed genes were obtained for each of the three stages relative to healthy individuals (Figure 1). The results obtained from the primary microarray analysis were further analyzed. Genes were identified that are differentially expressed (fold change $\geq 2$ or $\leq 0.5)$ specifically in each of Stage 2 (403 genes), Stage 3 (612 genes) and Stage 4 (31 genes) $\mathrm{CKDu}$. Genes that are commonly differentially expressed in stage 2 and 3 (303 genes), stage 3 and 4 (18 genes), and all the three stages ( 7 genes) were also identified. The seven genes commonly differentially expressed in all of the three stages have functions related to hypertensive response (ADM), gap junction channel activity (GJB4) and infectious/immune response (IFIT1, PI3, DEFA1, HBZ, RN7SK).

A)

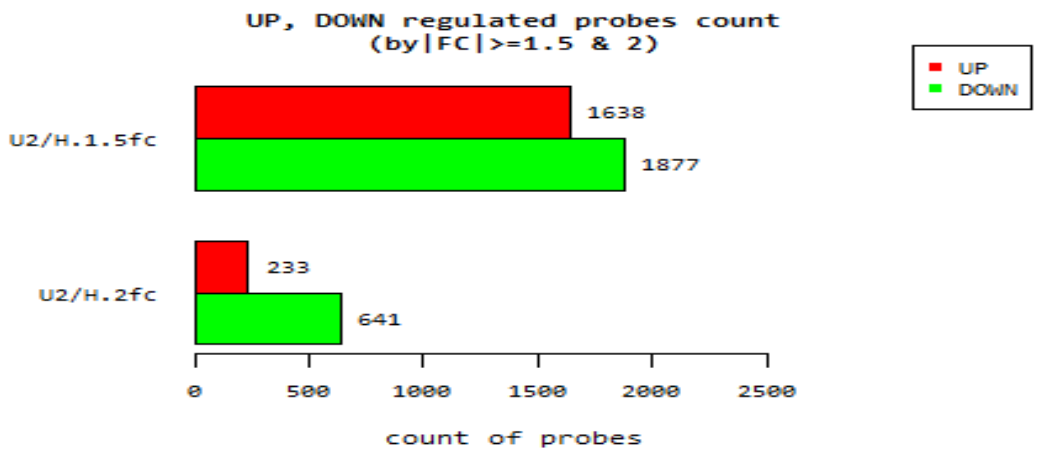


B)

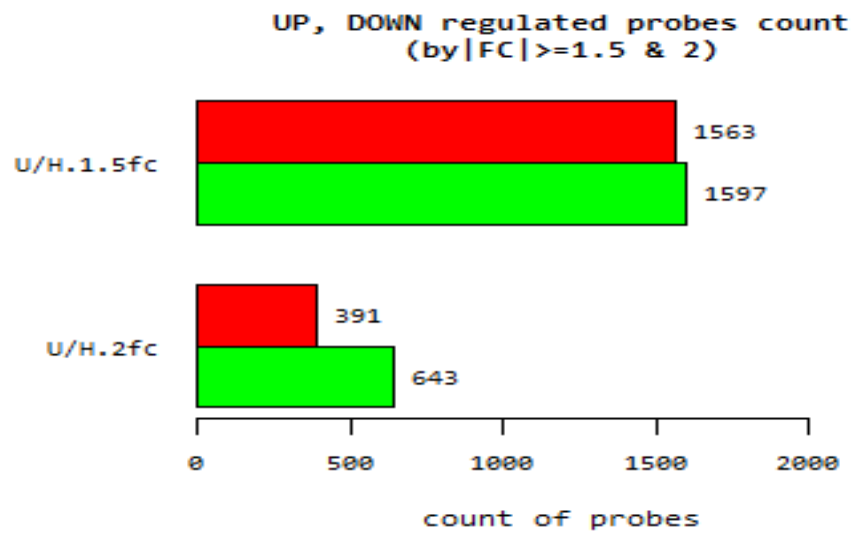

$=$ UP
= DOWN

C)

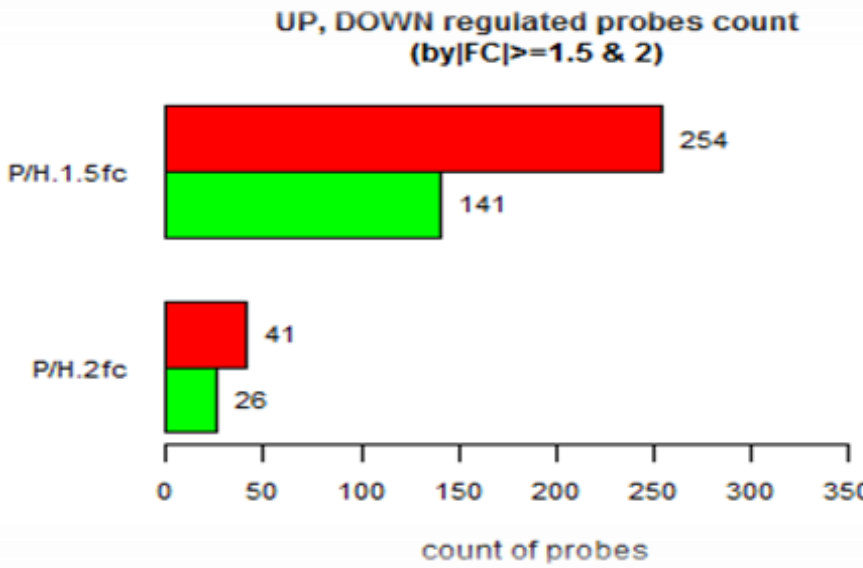

- up

= DOWN

Figure 1. Differentially expressed genes with fold change $(F C) \geq 1.5 \& 2$ for A) Stage 2 B) Stage 3 and C) Stage 4 CKDu 


\section{DISCUSSION}

From the transcriptome analysis of the three stages of $\mathrm{CKDu}$, seven genes from different functional groups were identified, that were commonly expressed in the diseased patients when compared to healthy individuals of a CKDu non-endemic area.

Adrenomedullin (ADM) is a potent hypotensive peptide having main functions of vasodilation, hypotension, angiogenesis, and regulation of fluid and electrolyte homeostasis $[9,10]$. The protein has been linked to considerable numbers of diseases, such as hypertension, congestive heart failure, ischemic heart injury, pulmonary hypertension, sepsis, cancers, renal impairment, and diabetes. Elevated plasma levels of ADM have been useful in assessing the progression of these diseases and circulating ADM was also seen to be increased after tissue transplantation, suggesting its protective role against oxidative damage. ADM holds high potential and is currently undergoing clinical studies to be used in diagnosis and treatment [10].

Gap junction protein beta 4 (GJB4) is coding for the protein Connexin 30.3 (Cx30.3) which is crucial for epidermal differentiation. Connexins are membranespanning proteins that allow for the formation of cellto-cell channels and cell-to-extracellular space hemi channels. Many connexin subtypes are expressed in kidney cells. Some mutations in connexin genes have been linked to various human pathologies, including cardiovascular, neurodegenerative, lung, and skin diseases, but the exact role of connexins in kidney disease remains unclear [11, 12]. Mutations of the GJB4 gene have been implicated in erythrokeratoderma variabilis with erythema gyratem repens $[12,13]$. Interestingly keratosis is a common symptom in CKDu patients and has been linked to arsenic poisoning [5].

Phosphatidylinositol 3-kinases (PI3K's), a family of enzymes which catalyze the phosphorylation of the $30-\mathrm{OH}$ of the inositol ring, play a central role in regulating a wide range of cellular processes including metabolism, survival, motility and cell activation. There is currently an intense focus by pharmaceutical companies to develop PI3K inhibitors as therapy for cancer, cardiovascular, respiratory, autoimmune and inflammatory diseases [14].
A few genes that were differentially expressed in the three stages of CKDu have previously been associated with viral and bacterial infections. The human Interferon-induced protein with Tetratricopeptide Repeats 1 (IFIT1) blocks West Nile virus (WNV), Japanese encephalitis virus (JEV), and coronavirus mutants $[15,16]$. The 7SK small nuclear RNA (RN7SK) functions as a negative regulator of HIV-1 transcription by interacting with the positive elongation factor $\mathrm{b}$ (p-TEFb) [17] . The upregulation of the Human T-cell leukemia virus type 1 (HTLV-1) basic leucine zipper factor gene (HBZ) has been associated with HTLV-1, the cause of Adult T-cell leukemia/lymphoma (ATL) [18, 19]. The HBZ gene was also suggested to possibly have a functional role in cellular transformation and leukemogenesis [19]. Human alpha-defensins like the alpha defensin 1 (DEFA1) have been reported as natural antimicrobial peptides of neutrophils which have evolved in host defense reactions. The circulating non stressed alphadefensins have also been associated with serum lipid levels [20].

From the results it can be seen that CKDu shares certain molecular level similarities to CKD of known origin and other metabolic disorders as is seen with the differential regulation of the ADM, GJB4, PI3K and DEFA1 genes. There also is a possibility of infections in this population as seen by the differential expression of the IFIT1, RN7SK, and HBZ genes. Real time quantitative PCRs are currently being carried out to verify findings of the microarrays. The regulation patterns of these genes need to be further looked into in detail in each of the stages to understand progression of the disease and for further biomarker development.

\section{CONCLUSION}

The seven identified genes; ADM, GJB4, PI3K, DEFA1, IFIT1, RN7SK and HBZ, together as a panel have potential as biomarkers of $\mathrm{CKDu}$, however need to undergo further validation studies prior.

\section{ACKNOWLEDGMENT}

The study was supported by the grant 11-059 received from the National Research Council, Sri Lanka. The corresponding author for the paper, D. N. 
Magana-Arachchi can be contacted via e mail at cellbio@ifs.ac.lk .

\section{REFERENCES}

[1]. Hamer RA, El Nahas AM: The burden of chronic kidney disease: Is rising rapidly worldwide. BMJ : British Medical Journal 2006, 332(7541):563-564.

[2]. Johnson S, Misra S, Sahu R, Saxena P: Environmental contamination and its association with $\mathrm{CKDu}$ in North Central Region of Sri Lanka. In. New Delhi, India: Centre for Science and Environment; 2012.

[3]. Weiner DE, McClean MD, Kaufman JS, Brooks DR: The Central American epidemic of CKD. Clinical journal of the American Society of Nephrology : CJASN 2013, 8(3):504-511.

[4]. Nanayakkara S, Komiya T, Ratnatunga N, Senevirathna ST, Harada KH, Hitomi T, Gobe G, Muso E, Abeysekera T, Koizumi A: Tubulointerstitial damage as the major pathological lesion in endemic chronic kidney disease among farmers in North Central Province of Sri Lanka. Environmental health and preventive medicine 2012, 17(3):213-221.

[5]. Nanayakkara S, Senevirathna ST, Abeysekera T, Chandrajith R, Ratnatunga N, Gunarathne ED, Yan J, Hitomi T, Muso E, Komiya T et al: An integrative study of the genetic, social and environmental determinants of chronic kidney disease characterized by tubulointerstitial damages in the North Central Region of Sri Lanka. Journal of occupational health 2014, 56(1):28-38.

[6]. Nanayakkara S, Senevirathna ST, Parahitiyawa NB, Abeysekera T, Chandrajith R, Ratnatunga N, Hitomi T, Kobayashi H, Harada KH, Koizumi A: Whole-exome sequencing reveals genetic variants associated with chronic kidney disease characterized by tubulointerstitial damages in North Central Region, Sri Lanka. Environmental health and preventive medicine 2015, 20(5):354-359.

[7]. Sayanthooran S, Magana-Arachchi D, Sooriyapathirana S, Abeysekera T, Gunarathne L: environmentally Influenced Gene Expression in Patients with Chronic Kidny Disease Living in the Dry Zone of Sri Lanka. 2014.

[8]. Cheung VG, Spielman RS: Genetics of human gene expression: mapping DNA variants that influence gene expression. Nature reviews Genetics 2009, 10(9):595-604.

[9]. Geny B, Ellero B, Charloux A, Brandenberger G, Doutreleau S, Piquard F: Circulating adrenomedullin is increased in relation with increased creatinine and atrial natriuretic peptide in liver-transplant recipients. Regulatory Peptides 2003, 114(1):61-66.

[10]. Ogoshi M: Adrenomedullin. 2016:240-e227D-244.

[11]. Lai-Cheong JE, Arita K, McGrath JA: Genetic diseases of junctions. The Journal of investigative dermatology 2007, 127(12):2713-2725.

[12]. Veenstra RD: Biology of Gap Junctions. 2012:409429.

[13]. Richard G, Brown N, Rouan F, Van der Schroeff JG, Bijlsma E, Eichenfield LF, Sybert VP, Greer KE, Hogan P, Campanelli $\mathrm{C}$ et al: Genetic heterogeneity in erythrokeratodermia variabilis: novel mutations in the connexin gene GJB4 (Cx30.3) and genotype-phenotype correlations. The Journal of investigative dermatology 2003, 120(4):601-609.

[14]. Foster JG, Blunt MD, Carter E, Ward SG: Inhibition of PI3K signaling spurs new therapeutic opportunities in inflammatory/autoimmune diseases and hematological malignancies. Pharmacological reviews 2012, 64(4):10271054 .

[15]. Danish H, Goyal S, Taunk NK, Wu H, Moran MS, Haffty BG: Interferon-induced Protein with Tetratricopeptide Repeats 1 (IFIT1) as a Prognostic Marker for Local Control in T1-2 N0 Breast Cancer Treated with Breast Conserving Surgery and Radiation Therapy (BCS+RT). International Journal of Radiation Oncology*Biology*Physics 2011, 81(2):S214.

[16]. Roby JA, Clarke BD, Khromykh AA: Loop de loop: viral RNA evades IFIT1 targeting. Trends in microbiology 2014, 22(4):171-173.

[17]. Krueger BJ, Varzavand K, Cooper JJ, Price DH: The mechanism of release of P-TEFb and HEXIM1 from the 7SK snRNP by viral and cellular activators includes a conformational change in 7SK. PloS one 2010, 5(8):e12335.

[18]. Landry S, Halin M, Vargas A, Lemasson I, Mesnard JM, Barbeau B: Upregulation of human T-cell leukemia virus type 1 antisense transcription by the viral tax protein. Journal of virology 2009, 83(4):2048-2054.

[19]. Matsuoka M, Green PL: The HBZ gene, a key player in HTLV-1 pathogenesis. Retrovirology 2009, 6:71-71.

[20]. Li YX, Lin CQ, Shi DY, Zeng SY, Li WS: Upregulated expression of human alpha-defensins 1,2 and 3 in hypercholesteremia and its relationship with serum lipid levels. Human immunology 2014, 75(11):1104-1109. 